\title{
Inferring introduction routes of invasive species using approximate Bayesian computation on microsatellite data
}

 \\ ${ }^{1} E q u i p e$ 'Biologie des Populations en Interaction', UMR 1301 Interactions Biotiques et Santé Végétale, INRA-CNRS-Université de Nice- \\ Sophia Antipolis, Sophia Antipolis, France; ${ }^{2}$ School of Animal and Microbial Sciences, University of Reading, Whiteknights, Reading, \\ UK; ${ }^{3} I N R A$ UMR Centre de Biologie et de Gestion des Populations (INRA/IRD/Cirad/Montpellier SupAgro), Campus international de \\ Baillarguet, Montferrier-sur-Lez, France and ${ }^{4}$ Department of Epidemiology and Public Health, Imperial College, St Mary's Campus, \\ London, UK
}

\begin{abstract}
Determining the routes of introduction provides not only information about the history of an invasion process, but also information about the origin and construction of the genetic composition of the invading population. It remains difficult, however, to infer introduction routes from molecular data because of a lack of appropriate methods. We evaluate here the use of an approximate Bayesian computation (ABC) method for estimating the probabilities of introduction routes of invasive populations based on microsatellite data. We considered the crucial case of a single source population from which two invasive populations originated either serially from a single introduction event or from two independent
\end{abstract}

introduction events. Using simulated datasets, we found that the method gave correct inferences and was robust to many erroneous beliefs. The method was also more efficient than traditional methods based on raw values of statistics such as assignment likelihood or pairwise $F_{S T}$. We illustrate some of the features of our $A B C$ method, using real microsatellite datasets obtained for invasive populations of the western corn rootworm, Diabrotica virgifera virgifera. Most computations were performed with the DIYABC program (http://www1.montpellier.inra.fr/CBGP/diyabc/).

Heredity (2010) 104, 88-99; doi:10.1038/hdy.2009.92; published online 5 August 2009

Keywords: $\mathrm{ABC}$; model selection; biological invasion; dispersal pathway

\section{Introduction}

In biological invasions, a large proportion of the genetic variability of an invading population depends on the historical and demographic features of its introduction: the number and genetic composition of sources, the number of successive introduction events from each source, the number of introduced individuals, the number of intermediate introduction steps between the source and the invaded region, and the dynamics of demographic expansion after each introduction. Determining the routes of introduction-the geographic pathways of the propagules between the source and the invading populations-therefore, provides not only information about the history of the invasion process, but also information about the origin and construction of the genetic composition of the invading populations (Dlugosch and Parker, 2008).

Two methods are traditionally used to make inferences about introduction routes: (1) Direct methods based on interception data and/or historical records of the presence or absence of the species (for example the study of

Correspondence: $\operatorname{Dr} T$ Guillemaud, Equipe 'Biologie des Populations en Interaction', UMR 1301 Interactions Biotiques et Santé Végétale, INRACNRS-Université de Nice-Sophia Antipolis, 400 Route des Chappes, BP 167, Sophia Antipolis cedex 6903, France.

E-mail: guillem@sophia.inra.fr

Received 7 March 2009; revised 12 June 2009; accepted 22 June 2009; published online 5 August 2009
Argentine ant by Suarez et al., 2001) and (2) indirect methods based on genetic relationships between populations (for example Kolbe et al., 2004; Ciosi et al., 2008). Such indirect methods are usually based on the calculation of genetic distance (for example Goldstein et al., 1999), assignment-likelihood statistics (Rannala and Mountain, 1997) and parsimony networks (for example Voisin et al., 2005).

One important limitation of the indirect methods is that they do not adequately take into account the demographic and genetic stochasticity of the history considered. The number of introduced individuals, their likelihood of becoming established and the time between introduction and demographic expansion may all be considered as random variables able to adopt various values. In addition, for a given set of demographic parameters, chance strongly affects the genetic composition of the samples studied because (i) genetic drift since the foundation event may have affected the genetic composition of the source population; (ii) genetic bottlenecks often occur during the first few generations after introduction, because of the limited number of founders and the small size of the newly founded population; (iii) mutational events may occur at any stage and (iv) limited numbers of individuals are usually collected during the field sampling of populations for genetic analysis. The level of stochasticity in introduction histories is, therefore, generally high, likely to have profound consequences and may considerably decrease the validity of the results obtained by indirect genetic methods. 
A second general problem of these direct and indirect methods is that they do not allow probabilistic estimations of competing introduction scenarios (but see Gaggiotti et al., 2004). In practice, an introduction scenario is chosen because the data are more consistent with that scenario than with any other. It is, in a sense, a binary decisional process (accept or reject), in which the relative likelihoods or weights of alternative scenarios are not known.

We evaluated here a model-based Bayesian method taking into account the stochasticity of the demographic and genetic processes involved and making it possible to calculate the relative probabilities of competing introduction scenarios. The Bayesian nature of this method makes it possible to make use of prior historical, biological and genetic information about the system. It is also based on a stochastic model linking demography and genetics - the coalescent model of the genealogical process (Kingman, 1982; Hudson, 1990; Nordborg, 2001) — to provide a simple and efficient population genetic model of drift and mutation particularly useful for handling complicated evolutionary scenarios. Estimations associated with demographic and genetic models often imply a full-likelihood calculation, which is difficult for complex scenarios such as biological invasions. Approximate Bayesian computation (ABC) based on summary statistics (Beaumont et al., 2002) does not require likelihood computation, but only requires the ability to simulate datasets and makes it possible to handle large datasets, such as data for hundreds of individuals genotyped at tens of microsatellite loci. This method was recently successfully used to estimate the demographic parameters of various complex scenarios (Fagundes et al., 2007; Pascual et al., 2007) and for model selection (Miller et al., 2005; Pascual et al., 2007; Cornuet et al., 2008).

We used simulated datasets to evaluate the utility of the $\mathrm{ABC}$ method for inferring the correct introduction routes of invasive populations. We considered microsatellite-like datasets, as this category of highly variable molecular markers have proved successful for addressing questions relating to invasive species (for example Estoup et al., 2004; Miller et al., 2005; Fagundes et al., 2007; Pascual et al., 2007). We evaluated this method in the simple, but crucial case of two invasive populations strongly suspected to have originated from the same source population. The key issue is determining whether the invasive populations originated from two independent introduction events or serially from a single introduction. This simple situation is the basis for retracing more complex multipopulational introduction histories (for example Miller et al., 2005). We have illustrated certain features of our simulation-based study by applying the $\mathrm{ABC}$ method to a recently published dataset for invasive populations of the western corn rootworm (WCR), Diabrotica vigifera virgifera (Kim and Sappington, 2005; Miller et al., 2005; Ciosi et al., 2008).

\section{Materials and methods}

\section{Models of introduction}

We considered three models in which two invasive populations originate from the same source population. The dataset consist of genotypes at statistically indepen-
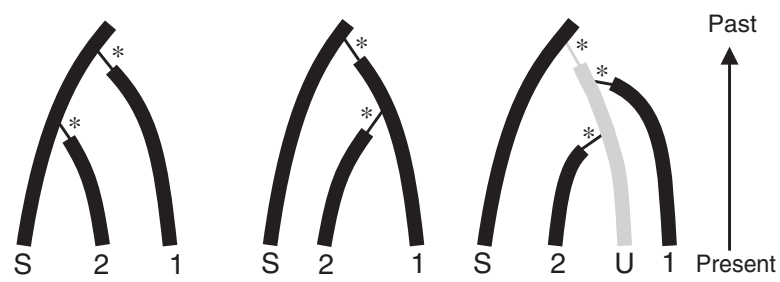

Independent introduction scenario

Serial introduction Unsampled population scenario scenario

* Bottleneck parameterization

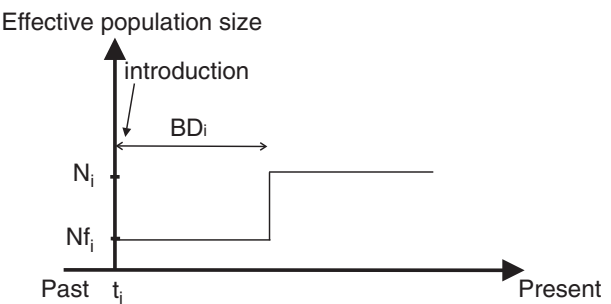

Figure 1 Introduction scenarios considered for the inference of the introduction routes of invading populations 1 and $2 . S$ is the source population in the native area, and $U$ is the unsampled population in the introduced area that is actually the source of populations 1 and 2 in the unsampled population scenario. The stars indicate the various bottlenecks occurring in the first few generations following introductions. The dynamics of these bottleneck events is represented in the lower part of the figure. $N f_{i}$ is the effective number of funders, $B D_{i}$ is the bottleneck duration and $N_{i}$ is the effective size of invading population $i$. Population $i$ was founded $t_{i}$ generations before present.

dent microsatellite loci obtained from a sample of diploid individuals collected from the invasive and source populations. These populations may be related through three different scenarios (see Figure 1).

The independent introduction scenario: Both introduced populations were founded independently from the source population. We assume that at each introduction, the invading population experienced a bottleneck because the number of founders is much lower than the number of individuals in a stable population. In this first scenario, each introduced population experienced a single bottleneck of possibly different intensities.

The serial introduction scenario: Only one introduced population originated from the source population, with the second introduced population originating from the first. The first introduced population, thus, experienced a single bottleneck (as in the independent introduction scenario), but the second introduced population experienced two successive bottlenecks, the first being common to both introduced populations.

The unsampled population scenario: Earlier studies have shown that some invasive populations may remain undetected, but may have important function in the invasion dynamics of some species (for example Saltonstall, 2002; Roman, 2006). In the two former scenarios, all the populations concerned were sampled, but in the unsampled population scenario, the two 
invasive populations were founded independently from an undetected and hence unsampled population, itself introduced from the source (Figure 1). Each invasive population experienced two bottlenecks, the first being common to both invasive populations. It is important to consider this scenario, because it superficially resembles an 'independent introduction scenario', with two introduced populations independently founded from a common population, but it actually corresponds to a single introduction from the native range. This is because only one introduced population, the unsampled population, originated from the native population. The variability of alleles and genetic combinations present in the invading populations is thus constrained by the genetic variation present in a single introduced population, rather than in two independently introduced populations. The likelihood of obtaining genetic combinations adapted to the new habitat is, therefore, lower than that in an independent introduction scenario. The 'unsampled population scenario' is hence both historically and evolutionarily substantially different from the independent introduction scenario.

Historical and demographic parameters were the same for all introduction models (see bottom part of Figure 1). Each introduced population $i$ was founded by individuals originating from its source population $t_{i}$ generations ago and was characterized by an effective number of founders, $N f_{i}$, remaining constant for a few generations (bottleneck duration $B D_{i}$ ) and then instantaneously reaching a larger stable effective population size, $N_{i}$. Parameters $t_{i}, N f_{i}$ and $B D_{i}$ can take distinct values in the various populations. In the meantime, the source population is assumed to have maintained a constant effective size $N_{s}$. The introduced populations are assumed to be isolated from each other and from the source population after the introduction, with no exchange of migrants. We also assume that no repeated introductions occurred at the same location.

\section{$A B C$ estimation of the posterior probabilities of scenarios} The posterior probabilities of competing scenarios were estimated with statistical methods implemented in the DIYABC program (Cornuet et al., 2008) available from http://www1.montpellier.inra.fr/CBGP/diyabc/. We simulated a large number (usually $3 \times 10^{5}$ ) of genetic datasets under the coalescent model (Kingman, 1982; Hudson, 1990; Nordborg, 2001), using the three introduction scenarios according to their prior probability and their parameter values drawn from prior distributions. Each typical genetic dataset contained the diploid genotypes, at 10 independent microsatellite loci, of 30 individuals sampled from each of the two invasive and the source populations. Diploid genotypes were generated assuming Hardy-Weinberg equilibrium by randomly grouping gene copies by pair within population. The summary statistics for each simulated dataset are recorded, together with the label of the scenario used for the simulation, in a file called the 'reference table'. As described by Beaumont et al. (2002), we calculated the Euclidean distances between each simulated dataset and the observed target dataset in the space of the summary statistics (standardized by the standard deviation of the simulated summary statistics) and these distances were then used to estimate the posterior probabilities of the scenarios. As we used simulation to evaluate the ABC method, the target 'observed datasets' were also simulated and will hereafter be referred to as 'pseudoobserved datasets'.

The posterior probabilities of the introduction scenarios were estimated by three methods. The first estimator, the 'direct estimator', is the frequency of Euclidean distances associated with scenario $i$ among distances below a specified threshold $z$ (Cornuet et al., 2008). The second estimator, derived from the $k$ th nearest-neighbor density estimator (equation 1.1 of Terrel and Scott, 1992), is called the 'KN estimator' and is defined as:

$$
P_{\mathrm{KN} i}=\frac{\left(\frac{1}{\delta_{i k}}\right)^{d}}{\sum_{i=1}^{n}\left(\frac{1}{\delta_{i k}}\right)^{d}}
$$

where $\delta_{i k}$ is the $k$ th smallest Euclidean distance for the introduction scenario $i$ and $d$ is the number of statistics used to summarize the data. As explained by Fagundes et al. (2007), the third estimator, the 'polychotomous logistic (PL) estimator', is based on the idea that we can sample the scenario indicator $i$ (where $i=1, \ldots, m$ for scenarios $1, \ldots, m$ ) from its prior and treat it as a categorical random variable in the ABC simulations. We can then apply a categorical regression (a PL regression) and an Epanechnikov kernel, as described by Beaumont (2008) to estimate the relative posterior probability of each scenario $i$. A proportion $y$ of the data points, corresponding to the smallest Euclidean distances, was used in the regression. Confidence intervals (CIs) of the PL estimator were computed as described in Cornuet et al. (2008).

The selection of suitable $z, k$ and $y$ thresholds is a difficult task, requiring cross validation procedures for each scenario tested. Low values of these thresholds result in the generation of accurate estimates, but high variances of the estimates. Large values may result in inaccurate estimates, but low variances of the estimates. We chose to use the lowest values ensuring both a small variance of the probability estimate among 20 reference tables and 1000 pseudo-observed datasets and good stability-that is weak variation of the estimates with variations of $z, k$ and $y$ (results not shown). The results obtained for $z$ and $k$ values between 50 and 1000 and for $y$ values between 1000 and 100000 (not shown) were all qualitatively similar to those presented below. A value of 100 for $z$ and $k$, and a value of 10000 for $y$ were hence selected for all calculations when not indicated differently.

\section{Summary statistics}

Genetic variation was summarized within and between populations, using the following statistics: the $F_{S T}$ values between pairs of populations (Weir and Cockerham, 1984) and the mean individual assignment likelihoods of individuals collected in population $i$ and assigned to population $j$ ( $L_{\mathrm{i} \rightarrow \mathrm{j}}$, Pascual et al., 2007), the mean number of alleles per locus, the expected heterozygosity $(H, \mathrm{Nei}$, 1987 ) and the mean variance of the absolute allelic size ( $V$, Estoup et al., 2004) computed for each population $(A$, $H, V$ ) or for each pair of populations (that is by pairwise pooling of population samples) $(A 2 P, H 2 P$ and $V 2 P)$. The default set of statistics (hereafter referred to as 'default stat') was $A 2 P, H 2 P, V 2 P, F_{S T}$ values and $L_{\mathrm{i} \rightarrow \mathrm{j}}$ and hence 
consisted of 18 statistics. We also used the set of statistics used by Miller et al. (2005) ('Miller stat': A, H, M (Garza and Williamson, 2001), $F_{S T}$ values and $L_{\mathrm{i} \rightarrow \mathrm{j}}$, which also correspond to a total of 18 statistics) and by Beaumont (2008) ('Beaumont stat': $A, H, V, A 2 P, H 2 P$ and $V 2 P$ for a total of 18 statistics). We compared the results obtained with 'default', 'Miller' and 'Beaumont stat' to select the best set of summary statistics.

\section{Prior distributions of parameters}

We kept our simulation study as generic as possible in the context of invasion biology. Each of the three competing scenario (independent, serial and unsampled) was assumed to be equally probable. The default set of prior distributions of the historical, demographic and mutational parameters is shown in Table 1. We used a generalized stepwise mutation model to simulate mutations at the molecular markers of interest (that is microsatellites; Estoup et al., 2002). A mean mutation rate across loci $\mu$ was first drawn from its distribution, then single locus mutation rates $\mu$ were drawn from a Gamma distribution with mean $\mu$ and shape parameter 2 (rate $=2 / \mu$ ). For each locus, the coefficient $P$ of the geometric distribution of repeat units by which a new mutant allele differs from its ancestor was drawn from an exponential distribution with mean of 0.22 (Miller et al., 2005).

\section{Simulation of pseudo-observed datasets}

For each introduction scenario, 1000 pseudo-observed genetic datasets were simulated using the DIYABC program as for the calculations above. The demographic, historical and mutational parameters used to simulate pseudo-observed data were drawn from probability distributions with regions of positive probabilities (that is supports) included within those of the prior distributions (Table 1). This assumption suggests that the knowledge about the biological system studied is sufficient to correctly choose the prior distributions. This procedure for simulating the test datasets was preferred over the more traditional strategy of fixing all but one of the parameters and evaluating the effect of the unfixed parameter, because the demographic, historical and mutational parameters were thought likely to act together to produce the pseudo-observed statistics and inference results. We used specific statistical treatments based on linear models to analyze the effect on posterior probabilities of varying the parameters used to simulate pseudo-observed datasets (see Supplementary information for details). For these analyses, we used a specific set of prior distributions: the 'alternative prior distributions' described in Table 1.

\section{Performance of the ABC approach}

The selected scenario was defined as the most probable of the scenarios considered. The performance of the ABC method was evaluated by measuring its accuracy. The accuracy of a classification method is the frequency at which it correctly selects the 'true' introduction scenario from among the tested scenarios. The term accuracy is used in the following with this meaning only. For each estimator, we also calculated the area under the curve (AUC) of a receiver operating characteristics graph (Fawcett, 2006). The largest AUC corresponds to the 
method giving the best compromise between true positive and false negative rates.

We evaluated the effect of the number of loci $(5,10,20$ and 50), sample size (15, 30 and 60 diploid individuals) and the number of simulated datasets in the reference table $\left(3 \times 10^{4}, 3 \times 10^{5}\right.$ and $\left.3 \times 10^{6}\right)$. The performance of the ABC method was also compared with that of indirect methods based on raw $F_{S T}$ or $L_{\mathrm{i} \rightarrow \mathrm{j}}$ statistics. With such methods, it is possible to infer introduction scenarios based on the following rules: the source of each introduced population is the sample for which the $L_{\mathrm{i} \rightarrow \mathrm{j}}$ value is the largest or the $F_{S T}$ value the smallest. Considering populations $S, 1$, and 2 in Figure 1, the independent introduction scenario $(S \rightarrow 1, S \rightarrow 2)$ is expected to produce the following relationships: $L_{1 \rightarrow S}>$ $L_{1 \rightarrow 2}, L_{2 \rightarrow \mathrm{S}}>L_{2 \rightarrow 1}$ and $F_{S T 12}>F_{S T S 1}, F_{S T 12}>F_{S T S 2}$. The serial introduction scenario $(S \rightarrow 1 \rightarrow 2)$ is expected to produce the following relationships: $L_{2 \rightarrow 1}>L_{2 \rightarrow \mathrm{S}}, L_{1 \rightarrow \mathrm{S}}$ $>L_{1 \rightarrow 2}$ and $F_{S T S 2}>F_{S T 12}, F_{S T S 2}>F_{S T S 1}$. No specific hierarchical relationship is expected for the unsampled population scenario.

\section{Robustness of inferences}

The robustness of the ABC method was evaluated by analyzing the effect of various erroneous beliefs on the biological system studied. Such errors were investigated by altering either the scenario or the distributions of parameters used to simulate the pseudo-observed datasets (that is the true introduction scenario and true parameter distributions). The prior parameter distributions and the three competing scenarios (independent, serial and unsampled) used for the inference remained unmodified. The following list of tests presents the various modifications of the true scenario and the true parameter distributions.

Test 1: The supports (that is, the regions of positive probabilities) of the true parameter distributions used to generate the pseudo-observed datasets were not included in those of the prior distributions (Table 1).

Test 2: False source in the invaded area: in the true introduction scenario, an introduced unsampled population has generated two serially introduced populations (unsampled-serial introduction scenario in Figure 2).

Test 3: False source in the native area: in the true introduction scenario, the sampled population in the native area had actually diverged from the real source

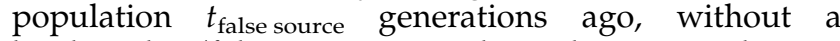
bottleneck (false source independent introduction scenario in Figure 2).

Test 4: Two sources: in the true introduction scenario, the two invasive populations were founded from two sources that had diverged $t_{\text {sources }}$ generations ago, and the source of only one invading population was sampled (two sources introduction scenario in Figure 2).

Test 5: False sequence of introductions: the sequence of introduction events was inverted because of uncertain, if not erroneous information concerning the dates on which the species was first sighted. The true inverted-serial introduction scenario (Figure 2) was hence source $\rightarrow$ pop $2 \rightarrow$ pop 1 , whereas the tested-serial



Figure 2 Introduction scenarios considered in tests 2, 3, 4 and 5 of the 'robustness analysis'. Test 2: false source in the introduced area (the unsampled-serial introduction scenario is shown as example). Test 3: false source in the native area (the false source independent introduction scenario is shown as example). Test 4: two sources. Test 5: false sequence of introduction (the inverted-serial introduction scenario is shown as example). $\mathrm{S}$ is the source population in the native area; $\mathrm{U}$ is the introduced unsampled population; FS is the false source in the native area; S1 and S2 are the two sources in the native area of the two invasive populations (in the example shown, only S2 was sampled). The gray color indicates that the corresponding population was not sampled.

scenario was source $\rightarrow$ pop $1 \rightarrow$ pop 2 . In the same vein, the true inverted independent introduction scenario was source $\rightarrow$ pop 2 and source $\rightarrow$ pop1, whereas the testedserial scenario was source $\rightarrow$ pop1 and source $\rightarrow$ pop 2 .

The prior distributions for the parameters used for tests 2, 3, 4 and 5 were those of the default set (Table 1), whereas $t_{\text {false source }}$ and $t_{\text {sources }}$ were drawn from Uniform[40;500].

\section{Application to the WCR, a pest beetle invading Europe} The WCR, D. virgifera Leconte, is a univoltine chrysomelid and is a major pest of corn in North America. It has recently been introduced into Europe. Several disconnected invading populations have been observed in Europe, including two large expanding outbreaks first observed in Serbia in 1992 (hereafter referred to as the Central European outbreak), and in North Western (NW) Italy in 2000. Miller et al. (2005) studied the introduction routes of WCR, using an earlier version of the ABC method (using the $\mathrm{KN}$ and direct estimators) and concluded that these two large expanding outbreaks were independently founded by individuals originating from North America. Here, we check this result using the revised $A B C$ method and, most importantly, we aim to illustrate the effect of considering erroneous source populations. We performed an ABC analysis on the NW Italian and Central European outbreaks, which were sampled in 2003 and described by Miller et al. (2005), using various North American samples as putative sources (samples collected in Texas and Pennsylvania in 2004, in Kansas, Nebraska, Iowa, Ohio, Illinois, Delaware and New York in 2003 (Kim and Sappington, 2005), and in Arizona in 1998 (Ciosi et al., 2008)). These American samples displayed low to medium levels of genetic differentiation ( $F_{S T}$ values ranging from 0 within the US Corn Belt to about 0.06 between Delaware and Arizona). We used the genotypes obtained at eight microsatellite loci (Miller et al., 2005). 
Table 2 Performance of the $\mathrm{ABC}$ and summary statistic-based methods

\begin{tabular}{|c|c|c|c|c|c|c|c|c|c|c|c|c|c|}
\hline \multirow[t]{3}{*}{ True scenario } & \multirow[t]{3}{*}{$\begin{array}{l}\text { Tested } \\
\text { scenarios }\end{array}$} & \multirow{2}{*}{\multicolumn{2}{|c|}{$\begin{array}{c}\begin{array}{c}\text { Summary } \\
\text { statistic } \\
\text { methods }\end{array} \\
\mathrm{f}\end{array}$}} & \multicolumn{10}{|c|}{$A B C$ method } \\
\hline & & & & \multicolumn{3}{|c|}{$\mathrm{f}$} & \multirow{2}{*}{$\begin{array}{l}\mathrm{f}_{l i m} \\
P L\end{array}$} & \multicolumn{3}{|c|}{$\overline{\mathrm{P}}$ (s.d.) } & \multicolumn{3}{|c|}{$\mathrm{f}\left(\mathrm{P}_{\mathrm{i}}>0.9\right)$} \\
\hline & & $\mathrm{F}_{\mathrm{ST}}$ & $\mathrm{L}_{i \rightarrow j}$ & Direct & $K N$ & $P L$ & & Direct & $K N$ & $P L$ & Direct & $K N$ & $P L$ \\
\hline \multirow[t]{3}{*}{ Independent } & Independent & 0.97 & 0.98 & 0.92 & 0.92 & 0.89 & 0.88 & $\begin{array}{c}0.77(0.19) \\
{[0.039]}\end{array}$ & $\begin{array}{c}0.86(0.21) \\
{[0.027]}\end{array}$ & $\begin{array}{c}0.81(0.22) \\
{[0.016]}\end{array}$ & 0.26 & 0.65 & 0.50 \\
\hline & Serial & 0.01 & 0.01 & 0.00 & 0.00 & 0.00 & 0.00 & $\begin{array}{c}0.02(0.05) \\
{[0.009]}\end{array}$ & $\begin{array}{c}0.01(0.05) \\
{[0.003]}\end{array}$ & $\begin{array}{c}0.01(0.03) \\
{[0.001]}\end{array}$ & 0 & 0 & 0 \\
\hline & Unsampled & $\mathrm{NC}$ & $\mathrm{NC}$ & 0.08 & 0.08 & 0.11 & 0.09 & $\begin{array}{c}0.21(0.17) \\
{[0.038]}\end{array}$ & $\begin{array}{c}0.13(0.20) \\
{[0.027]}\end{array}$ & $\begin{array}{c}0.18(0.21) \\
{[0.016]}\end{array}$ & 0 & 0.10 & 0.10 \\
\hline \multirow[t]{3}{*}{ Serial } & Independent & 0.03 & 0.00 & 0.00 & 0.00 & 0.00 & 0.00 & $\begin{array}{c}0.00(0.03) \\
{[0.003]}\end{array}$ & $\begin{array}{c}0.00(0.03) \\
{[0.001]}\end{array}$ & $\begin{array}{c}0.00(0.02) \\
{[0.000]}\end{array}$ & 0 & 0 & 0 \\
\hline & Serial & 0.94 & 0.37 & 0.91 & 0.92 & 0.91 & 0.90 & $\begin{array}{c}0.72(0.15) \\
{[0.044]}\end{array}$ & $\begin{array}{c}0.81(0.18) \\
{[0.045]}\end{array}$ & $\begin{array}{c}0.79(0.17) \\
{[0.016]}\end{array}$ & 0.08 & 0.45 & 0.34 \\
\hline & Unsampled & $\mathrm{NC}$ & $\mathrm{NC}$ & 0.08 & 0.08 & 0.09 & 0.06 & $\begin{array}{c}0.28(0.14) \\
{[0.044]}\end{array}$ & $\begin{array}{c}0.18(0.18) \\
{[0.045]}\end{array}$ & $\begin{array}{c}0.21(0.17) \\
{[0.016]}\end{array}$ & 0 & 0 & 0 \\
\hline \multirow[t]{3}{*}{ Unsampled } & Independent & 0.37 & 0.24 & 0.01 & 0.01 & 0.01 & 0.01 & $\begin{array}{c}0.04(0.10) \\
{[0.011]}\end{array}$ & $\begin{array}{c}0.03(0.10) \\
{[0.006]}\end{array}$ & $\begin{array}{c}0.02(0.08) \\
{[0.002]}\end{array}$ & 0 & 0 & 0 \\
\hline & Serial & 0.29 & 0.15 & 0.05 & 0.06 & 0.03 & 0.03 & $\begin{array}{c}0.17(0.16) \\
{[0.033]}\end{array}$ & $\begin{array}{c}0.10(0.18) \\
{[0.026]}\end{array}$ & $\begin{array}{c}0.14(0.14) \\
{[0.011]}\end{array}$ & 0 & 0 & 0 \\
\hline & Unsampled & $\mathrm{NC}$ & $\mathrm{NC}$ & 0.93 & 0.93 & 0.96 & 0.94 & $\begin{array}{c}0.79(0.16) \\
{[0.039]}\end{array}$ & $\begin{array}{c}0.87(0.19) \\
{[0.032]}\end{array}$ & $\begin{array}{c}0.84(0.15) \\
{[0.013]}\end{array}$ & 0.28 & 0.66 & 0.47 \\
\hline Mean accuracy & & 0.64 & 0.45 & 0.92 & 0.92 & 0.92 & 0.91 & & & & & & \\
\hline
\end{tabular}

Direct, KN and PL represent three different estimation methods of posterior probabilities of introduction scenarios as described in the Materials and methods section. Indirect methods are based on raw $F_{S T}$ or assignment likelihood $L_{i \rightarrow j}$ values. $f$ is the proportion of the simulated data classified into each tested scenario. The frequency at which the lower limit of the $95 \%$ confidence interval of the posterior probability of each scenario exceeds the upper limit of alternative scenarios is $f_{\text {lim }}$ (computation possible only for the PL estimator). For the ABC method, the mean $(\bar{P})$ and the standard deviation (s.d.) of the posterior probability of each tested scenario and the frequency of posterior probabilities exceeding $0.9\left(f\left(P_{i}>0.9\right)\right)$ are shown. In addition, the mean standard error of the posterior probability of each tested scenario among 30 reference tables is given in square brackets. Values in bold characters indicate that the corresponding tested scenario is true. NC is not computable.

The default set of priors detailed in Table 1 was used, with the following minor modifications: the years of introduction of WCR in Europe were drawn from uniform distributions bounded by 1986 and 1991 for Central Europe, and by 1995 and 1999 for the NW Italian outbreak. The generation time for $D$. virgifera is 1 year. Given the available data on the fertility and population growth rates of WCR (Toepfer and Kuhlmann, 2005), we uniformly draw duration of bottlenecks after the introduction between 1 and 5 years, as in the study by Miller et al. (2005).

\section{Results}

\section{Inferring introduction scenarios}

We found the $\mathrm{ABC}$ method for inferring the introduction routes of invading populations to be efficient in most of the simulations studied, whatever the estimator of the posterior probabilities (Table 2). When scenario selection was based on the highest posterior probability value, between 89 and $96 \%$ of the pseudo-observed datasets were correctly assigned to the true scenario (mean accuracy of 0.92). Note that with this classification criterion, a classification can be correct, although the estimated probability of the true scenario is below 0.9. This explains why the frequency of correct classification ( $f$ in Table 2 ) is generally high, although $f\left(P_{i}>0.9\right)$ may be low. The unsampled population scenario was well recovered by the ABC method.
The traditional indirect method (described in the 'Performance of the ABC approach' section), in which raw $F_{S T}$ values are used to infer the introduction scenario, correctly classifies the pseudo-observed datasets to the independent or serial scenario (Table 2). It is worth stressing, however, that this method cannot correctly classify the unsampled population scenario and selects the independent or serial introduction scenario for $66 \%$ of the datasets simulated under the unsampled population scenario. The method based on mean individual assignment likelihood did not provide satisfactory results. More than $60 \%$ of the pseudoobserved data simulated under the serial introduction scenario could not be assigned to either the independent or serial introduction scenario. Similar to the $F_{S T}$-based method, the assignment-likelihood method cannot classify the unsampled population scenario. It is noteworthy that when an introduction scenario is incorrectly selected, the differences between assignment likelihoods or between $F_{S T}$ values whose relationships are used to infer the scenario are lower than when the scenario is correctly selected. For instance the differences $L_{1 \rightarrow \mathrm{S}}-L_{1 \rightarrow 2}, L_{2 \rightarrow \mathrm{S}}-L_{2 \rightarrow 1}, F_{S T 12}-F_{S T S 1}, F_{S T 12}-F_{S T S 2}$ are larger if the independent scenario is correctly selected than if it is incorrectly selected (for example when the unsampled population scenario is true).

Additional analyses showed that when the bottleneck population sizes used to simulate the pseudo-observed datasets were larger than in the default conditions, the simple indirect methods performed much less well than 
the ABC method. With bottleneck population sizes drawn from a uniform prior distribution between 50 and 500 and from a uniform distribution to generate the pseudo-observed datasets bounded by 100 and 500 (all other distribution being the same as before), we obtained the following frequencies of correct scenario identification: $67.4 \%$ for the $F_{S T}$ method, $70 \%$ for $L_{i \rightarrow j}$ and $83.6 \%$ for $\mathrm{ABC}$ when the true scenario was the independent scenario; and $65.3 \%$ for the $F_{S T}$ method, $33.1 \%$ for $L_{i \rightarrow j}$ and $80.4 \%$ for $\mathrm{ABC}$ when the serial scenario was true. As already mentioned, the unsampled population scenario could not be identified by the $F_{S T}$ and $L_{i \rightarrow j}$ methods. It was correctly identified by the ABC method at a rate of $53.9 \%$.

Comparing estimators of introduction scenario probability The expected values of the posterior probabilities of our complex competing scenarios are unknown, but a 'good' estimator should frequently assign the pseudo-observed datasets to the introduction scenario used to simulate them. We found that the three estimators behaved very similarly in this respect (Table 2).

Another desirable property of the posterior probability estimator is its small variance among independent analyses of the same data. The standard error of the posterior probability estimator was calculated by analyzing the 3000 simulated pseudo-observed datasets 30 times, with 30 independent reference tables. The PL estimator had a standard error between reference tables one-half to one-third that of the other estimators (see the values in brackets in Table 2).

Importantly, as mentioned in the Materials and methods section, the PL estimator displayed a remarkable stability - that is weak variation of the estimates with variations of $y$, the proportion of the data points used in the regression. We observed almost no variation of PL estimates with $y$ values varying between 1000 and 100000.

Finally, we could calculate CIs for the posterior probability of the PL estimator. Use of the lower limit of the $95 \%$ CI of the maximal probability scenario being greater than the upper limit of the $95 \%$ CI of the other scenarios as the classification criterion gave an accuracy similar to that obtained with the largest posterior probability (Table 2). The overall properties of the PL estimator were thus considered as better than those of the direct and KN estimators. We, therefore, present only the results obtained with the PL estimator below.

\section{Effects of model parameter values on inferences}

The alternative parameter distributions and alternative prior distributions (Table 1) used to address this particular question provided good discrimination results: $\mathrm{AUC}=0.99$, accuracy $=0.95$.

We performed a statistical analysis of the effects of variation in the parameters on the posterior probabilities of scenarios, using a linear model-based approach. The results of the linear model fits are shown in Supplementary Table 1. Linear models accounted for 22, 31 and $45 \%$ of the variance when the true scenario was the independent, serial or unsampled population scenario, respectively. Most significant and larger effects dealt with bottleneck parameters: the posterior probabilities of the independent and serial scenario, when true, were negatively correlated with the intensity of drift in the two introduced populations during bottleneck events. The probability of the true scenario was higher for larger bottleneck population sizes and shorter bottleneck durations. When the unsampled population scenario was the true scenario, the effect of drift intensity in the introduced populations depended on the population considered. Drift intensity had a negative effect in the more recent introduced population (population 2 in Supplementary Table 1), and a variable effect in the older introduced population (population 1) and in the unsampled population. Increasing the number of effective founders in the more recently introduced and unsampled populations had a positive effect on the probability of the true scenario below a certain population size and a negative effect above this threshold. Parameters affecting genetic diversity in the source population (that is $N_{\text {source }}$ and $\bar{\mu}$ ) had a positive effect on the posterior probability of the independent and serial introduction route scenarios, but no significant effect for the unsampled population scenario. The dates of introductions and stable effective population sizes after the bottleneck period, for introduced populations, had no effect on the posterior probability for any of the scenarios.

\section{Influence of $A B C$ summary statistics, number} of individuals, loci and reference table size

The number of datasets in the reference table, the number of diploid individuals sampled per population and the number of loci have a positive effect on the AUC, the probability of the true scenario and the rate of identification of the true scenario (Table 3). The ABC method was found to be sensitive to the number of loci used, but provided good results with as few as five loci ( $>70 \%$ of scenarios correctly identified). Reducing the sampling effort to 15 individuals per sample decreased the accuracy by about 5 percentage points with respect to the default sample size (30 individuals). Doubling the default sample size per population did not increase accuracy. The use of $3 \times 10^{6}$ datasets per reference table gave no improvement in the correct classification rate over the use of $3 \times 10^{5}$ datasets, and $3 \times 10^{4}$ datasets appear sufficient to reach a similar accuracy. Finally, all tested sets of ABC summary statistics ('default', 'Miller', 'Beaumont stat') gave similar results, with an accuracy of between 91 and $92 \%$ (Table 3).

\section{Robustness of inference and effect of erroneous prior beliefs}

Test 1: Errors in demographic, historical and genetic parameter priors (Table 4): using priors for $N_{s}$ with larger values than that of $N_{s}$ used to simulate pseudo-observed datasets decrease the posterior probabilities of the true scenarios and increases their variances. Consequently, the correct introduction scenario is recovered less often, even if it is correctly inferred in $>70 \%$ of the simulated cases. Conversely, if the priors used for $N f_{i}$ are too small, the rate of correct classification increases markedly when the true scenario is the independent or serial scenario. The true unsampled population scenario is poorly recovered, however, and the distribution of the posterior probabilities of the three scenarios is generally flat in this case $(<7 \%$ of the probabilities 
Table 3 Effect of number of loci and individuals per sample, reference table size and ABC summary statistics

\begin{tabular}{|c|c|c|c|c|c|c|c|c|c|c|c|c|c|c|}
\hline \multirow[t]{3}{*}{ Parameters } & \multirow[t]{3}{*}{ AUC } & \multirow[t]{3}{*}{ Accuracy } & \multicolumn{12}{|c|}{ True introduction scenario } \\
\hline & & & \multicolumn{4}{|c|}{ Independent } & \multicolumn{4}{|c|}{ Serial } & \multicolumn{4}{|c|}{ Unsampled } \\
\hline & & & $\overline{\mathrm{P}}_{\text {indep }}($ s.d. $)$ & $\mathrm{f}_{\text {indep }}$ & $\mathrm{f}_{\text {serial }}$ & $\mathrm{f}_{\text {unsampled }}$ & $\overline{\mathrm{P}}_{\text {serial }}$ (s.d.) & $\mathrm{f}_{\text {indep }}$ & $\mathrm{f}_{\text {serial }}$ & $\mathrm{f}_{\text {unsampled }}$ & $\overline{\mathrm{P}}_{\text {ghost }}$ (s.d.) & $\mathrm{f}_{\text {indep }}$ & $\mathrm{f}_{\text {serial }}$ & $\mathrm{f}_{\text {unsampled }}$ \\
\hline Standard & 0.991 & 0.92 & $0.81(0.22)$ & 0.89 & 0.00 & 0.11 & $0.79(0.17)$ & 0.00 & 0.91 & 0.09 & $0.84(0.15)$ & 0.01 & 0.03 & 0.96 \\
\hline \multicolumn{15}{|l|}{ Number of loci } \\
\hline 5 & 0.967 & 0.85 & $0.73(0.25)$ & 0.81 & 0.01 & 0.18 & $0.72(0.19)$ & 0.00 & 0.85 & 0.15 & $0.77(0.18)$ & 0.03 & 0.07 & 0.89 \\
\hline 20 & 0.995 & 0.95 & $0.86(0.21)$ & 0.92 & 0.00 & 0.08 & $0.84(0.15)$ & 0.00 & 0.95 & 0.05 & $0.87(0.13)$ & 0.01 & 0.02 & 0.97 \\
\hline 50 & 0.999 & 0.98 & $0.93(0.16)$ & 0.96 & 0.00 & 0.04 & $0.88(0.12)$ & 0.00 & 0.98 & 0.02 & $0.90(0.10)$ & 0.00 & 0.01 & 0.99 \\
\hline \multicolumn{15}{|c|}{ Number of individuals } \\
\hline 15 & 0.978 & 0.87 & $0.73(0.24)$ & 0.83 & 0.01 & 0.16 & $0.73(0.19)$ & 0.00 & 0.87 & 0.13 & $0.79(0.17)$ & 0.01 & 0.07 & 0.92 \\
\hline 60 & 0.991 & 0.93 & $0.82(0.23)$ & 0.89 & 0.00 & 0.11 & $0.80(0.17)$ & 0.00 & 0.93 & 0.07 & $0.85(0.15)$ & 0.01 & 0.03 & 0.96 \\
\hline \multicolumn{15}{|c|}{ Number of datasets in reference table } \\
\hline $3 \times 10^{4}$ & 0.990 & 0.92 & $0.83(0.24)$ & 0.89 & 0.00 & 0.11 & $0.79(0.15)$ & 0.00 & 0.95 & 0.05 & $0.87(0.18)$ & 0.01 & 0.06 & 0.93 \\
\hline $3 \times 10^{6}$ & 0.990 & 0.92 & $0.81(0.23)$ & 0.88 & 0.00 & 0.12 & $0.79(0.16)$ & 0.00 & 0.94 & 0.06 & $0.85(0.17)$ & 0.01 & 0.05 & 0.94 \\
\hline \multicolumn{15}{|l|}{ Statistics } \\
\hline Miller & 0.990 & 0.92 & $0.80(0.22)$ & 0.89 & 0.00 & 0.11 & $0.79(0.17)$ & 0.00 & 0.92 & 0.08 & $0.83(0.16)$ & 0.01 & 0.05 & 0.94 \\
\hline Beaumont & 0.988 & 0.91 & $0.79(0.23)$ & 0.87 & 0.00 & 0.13 & $0.77(0.16)$ & 0.00 & 0.93 & 0.07 & $0.84(0.17)$ & 0.01 & 0.05 & 0.94 \\
\hline
\end{tabular}

The effects on inferences were evaluated by calculating the mean accuracy, the mean one-versus-all AUC of the classification, the mean $\left(\bar{P}_{i}\right)$ and the standard deviation (s.d.) of the posterior probability of the true scenario $i$, and the proportion of cases in which scenario $i$ has the largest posterior probability $\left(f_{i}\right)$. See Materials and methods section for details regarding default conditions. The default number of loci, individuals and datasets in the reference table are 10,30 and $3 \times 10^{5}$, respectively.

exceed 0.9 versus $47 \%$ for the default parameters; Table 2).

If bottleneck duration priors are too small, scenario identification becomes much less accurate, particularly if the serial scenario is true. In this situation, the unsampled population scenario is erroneously selected in $72 \%$ of the simulated cases. However, as stated earlier, the distributions of the posterior probabilities of the three scenarios are flat $(<10 \%$ of the probabilities exceed 0.9 versus $>30 \%$ for the default parameters; Table 2).

Erroneous beliefs concerning mutational parameters had limited effects on inference accuracy, unless the mean mutation priors are too large.

A large error in the timing of introduction events (such as the actual introductions taking place about 100 generations before they were believed to occur) had only a small effect on inferences. However, the unsampled population scenario tended to be selected more frequently when the serial introduction scenario was true than in the default situation (26 versus 9\%).

Test 2: False source in the invaded area: the $A B C$ method selects the serial scenario when the unsampledserial scenario is true with about the same frequency as when the serial scenario is true.

Test 3: False source in the native area: when the two populations were introduced independently only $39 \%$ of the simulated cases were assigned to the independent scenario and $61 \%$ falsely assigned to the unsampled population scenario. This effect depends on the level of differentiation between the actual and the believed source (see Supplementary Figure 1). Moderate to high levels of divergence $\left(F_{S T}>0.02\right)$ led to selection of the (false) unsampled population scenario. However, if only independent and serial scenarios are considered, the rate of recovery of the true scenario is high (that is $84.8 \%$ for independent and 100\% for serial scenarios; results not shown).

Test 4: Two sources: if the two invasive populations originate from two diverging sources (quartiles of $F_{S T}$ distribution: $0.008,0.014,0.021$ ), it is believed that one of these sources is the source of both populations (see 'Two sources' in Table 4), the independent scenario is recovered as frequently as in the default situation.

Test 5: False sequence of introductions: when the trueserial scenario is source $\rightarrow$ pop $2 \rightarrow$ pop 1 , but the tested scenario is source $\rightarrow$ pop $1 \rightarrow$ pop 2 , the unsampled population scenario is incorrectly selected in most cases (94\%). However, no effect on posterior probabilities was observed when the independent or the unsampled population scenario was the true scenario. This led us to consider this 'inverse-serial scenario' to the scenarios tested, resulting in the consideration of four, rather than three competing scenarios (that is independent, serial, inverse-serial and unsampled scenarios) in a dedicated analysis (Supplementary Table 2). The true inverse-serial scenario was correctly recovered in $91 \%$ of the simulated cases (Supplementary Table 2). Adding an alternativeserial scenario to the set of scenarios tested only slightly decreased the global accuracy of the ABC method (from 92 to $89 \%)$. For the sake of clarity, we considered this fourth (inverse serial) scenario in this specific analysis (Supplementary Table 2) only.

\section{Application to the WCR}

We performed 10 successive $\mathrm{ABC}$ analyses of introduction scenarios, using each of the 10 North American 
96



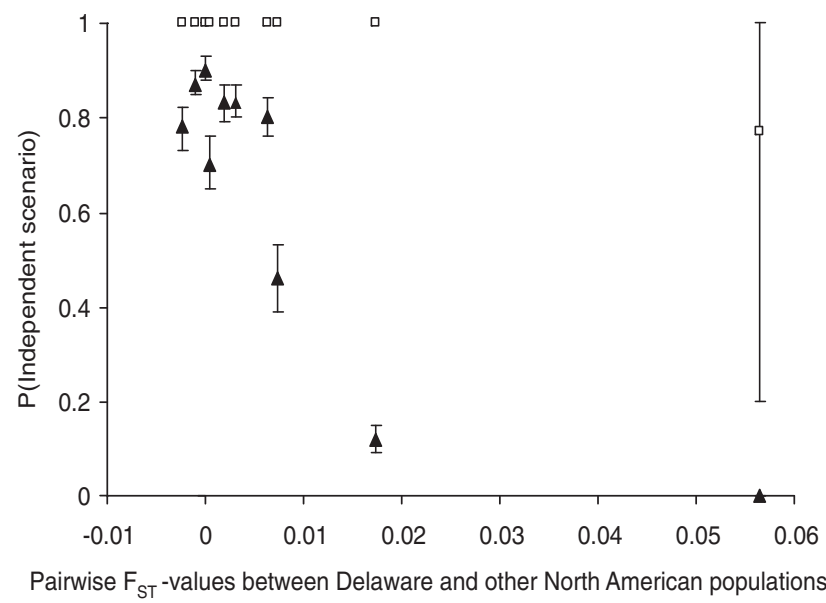

Figure 3 Posterior probability of the independent scenario in the case of the NW Italian and Central European WCR invasive outbreaks shown as a function of the $F_{S T}$ value between the various source populations used to compute the probability and the Delaware sample, considered to be the 'true' source of the European outbreaks (see text for details). The error bars correspond to the $95 \%$ $\mathrm{CI}$ of the PL regression estimator. The black triangles correspond to the case, in which three scenarios are tested (independent, serial and unsampled population), and the open squares to the case, in which only two scenarios are tested (independent and serial). The serial scenario has a null posterior probability, whatever the source population used and, thus, $P($ Unsampled $)=1-P($ Independent $)$.

samples as the source of the European populations. The serial scenario had a null posterior probability whatever the source population used. If one of the samples collected from the central and eastern part of the USA (from Kansas to the state of New York) was used as the source, the probability of the independent introduction scenario was high (between 0.7 and 0.9 with narrow 95\% CIs), except for the sample from Illinois, which gave a probability of 0.46 (95\% CI: $0.39-0.53)$. Interestingly enough, we found that the unsampled population scenario was selected with probabilities of 1 (95\% CI: $1-1)$ and 0.88 (95\% CI: 0.85-0.91) if we used the samples from Arizona and Texas, respectively, as the source.

For the sake of simplicity, we defined the 'true' source population of the European outbreaks as the North American population with the lowest $F_{S T}$ value with respect to the NW Italian and Central European samples. The sample collected in Delaware was thus considered the most probable source of both European populations. Figure 3 shows the relationships between the posterior probability of the independent scenario and the pairwise $F_{S T}$ values obtained by comparison between each 'false' source population used to compute the probability and the Delaware sample. Consistent with our earlier simulation results, there was a marked inverse correlation between the probability of the independent scenario and $F_{S T}$. Figure 3 also shows that when only two scenarios are tested (independent and serial introduction scenarios), the posterior probability of the independent scenario is 1, with a small $95 \%$ CI for all of the US samples considered as the source except for the Arizona sample. The ABC method correctly selects the unsampled population scenario, when true, whether or not beliefs concerning the source in the area of origin are erroneous (Table 4). Thus, our results for WCR strongly 
support the hypothesis of an independent introduction scenario with a source population within or close to the central or eastern part of the USA. They also nicely illustrate the potential bias that is likely to arise when considering a wrong genetically differentiated source population.

\section{Discussion}

We evaluated the ability of an ABC method to infer introduction routes in the context of biological invasions. Using a simulation-based approach, we showed that, when two invading populations and a source population are considered, the $\mathrm{ABC}$ method very frequently identifies the 'true' introduction scenario. This method also provides an estimate of the posterior probability of the competing scenarios and CIs for each probability. It is worth stressing that a set of $n$ invasive populations with a common source population in the native area can be analyzed by studying $n(n-1) / 2$ pairs of invasive populations with the independent, serial and unsampled population scenarios (Miller et al., 2005).

Calculation of the posterior probabilities for each competing scenario is of key importance, as it makes it possible to quantify our level of confidence in the choice of a specific scenario (as opposed to the others). The Bayesian nature of the $\mathrm{ABC}$ method confers on this method the advantage that prior information can be incorporated such that the competing scenarios have different weights. For instance, the dates of the first observations of introduced populations can be used to weight the serial and inverse-serial introduction scenarios, as proposed byMiller et al. (2005).

Methods based on $F_{S T}$ or $L_{\mathrm{i} \rightarrow \mathrm{j}}$ values to which the $\mathrm{ABC}$ approach was compared are not the most powerful (for example as compared with full-likelihood methods). However, 'distance' methods, some of which are tree based, are commonly used in invasion biology to determine the most probable sources of invasive populations and, by extension, to determine introduction routes of invasive populations. We found that $F_{S T}$ or $L_{\mathrm{i} \rightarrow \mathrm{j}}$ methods were less reliable than the $\mathrm{ABC}$ method for at least three reasons: (1) they cannot include the unsampled population scenario among the tested models; more generally, as soon as a tested scenario is not directly translatable into a simple hierarchy between $F_{S T}$ values or between $L_{\mathrm{i} \rightarrow \mathrm{j}}$ values, it cannot be evaluated. More importantly, when the unsampled population scenario is true, these methods erroneously identify either the independent or the serial scenario as the true scenario, (2) they result in incorrect classification when bottleneck intensities are moderate and, most importantly, (3) they do not provide probabilities for the introduction routes tested or any other measurement of confidence in the choice made. Many earlier studies in the field of invasion biology (e.g. Kolbe et al., 2004; Voisin et al., 2005) have been based on methods using distance trees or parsimonious networks built from nucleotide sequence data (often mitochondrial or chloroplast DNA). Drawbacks (1) and (3) of the $F_{S T}$ and $L_{i \rightarrow j}$ approaches probably also apply to these methods. A simulation-based study is required to evaluate drawback (2).

We compared three different estimators of posterior probabilities: the direct estimator, the $k$ th neighbor density estimator (KN) and the PL regression estimator.
We found that the PL estimator had desirable properties, such as a low sensitivity to the choice of threshold and a low variance. However, it often provided lower values for true scenario probabilities than the KN estimator. The reanalysis in this paper of some of the data of Miller et al. (2005) using the PL estimator confirmed, with high levels of confidence, that the NW Italian and Central European outbreaks of WCR resulted from independent introductions from North America (most probably from the central north-eastern part of the USA).

The default parameters used in our simulation settings correspond to an a priori unfavorable situation, in which the invasions were recent. In such conditions, genetic differentiation would be expected to be minimal and signatures of the introduction history because of long divergence times between populations should be absent. Consistent with these assumptions, we showed that bottleneck intensities were key factors for determining the posterior probabilities of introduction routes. We might initially have expected stronger bottleneck intensities to favor scenario discrimination, because drift pulses during bottlenecks are at least partly responsible for the genetic signature of introductions. However, we found the opposite to be true. Accuracy was negatively affected by bottleneck intensity, probably because intense bottlenecks (particularly during the first introduction event) tend to generate patterns expected under the unsampled population scenario, in which the gene genealogies of both introduced populations suffer from two successive bottlenecks.

We found that our approach was robust to many types of error in prior beliefs. In particular, errors concerning mutational parameters and the dates of events were found to have negligible effects on classification results. If only the independent and serial scenarios are considered, the ABC method is almost insensitive to error concerning demographic parameters, including bottleneck intensity. The unsampled population scenario may increase the susceptibility of the analysis to errors in prior beliefs, but including this scenario in the set of tested scenarios is crucial, as it avoids confusion between multiple and single introduction scenarios. It might be worth reanalyzing some of the earlier reported descriptions of multiple introduction scenarios that may actually be the result of unsampled population scenarios.

We found that in case of geographic genetic structure within the native area, an error concerning the source population in this area resulted in misleading results being obtained with the ABC method. The probability of the unsampled population scenario increases with the level of genetic differentiation between the true and false sources when the true scenario is an independent introduction scenario. We have illustrated this effect with real datasets from the WCR invasion in Europe. The NW Italian and Central European outbreaks of WCR probably resulted from independent introductions of individuals originating in the northern US (Miller et al., 2005; Ciosi et al., 2008). If we considered the source population to have originated from the central or northeastern USA, the independent scenario was selected with considerable support. However, if we considered samples from Texas or Arizona, genetically differentiated from the samples collected in central or north-eastern USA, to be the source, the unsampled population scenario was selected with high posterior probabilities. 
Again, if the unsampled population scenario is not considered among the tested scenarios, then the $A B C$ method is insensitive to errors concerning the source population. We also showed that in cases of uncertainty concerning the order of introduction events, a satisfactory solution is to include the inverse-serial scenario among the tested scenarios (as shown by Miller et al., 2005). Our study shows that this approach prevents the misclassification of serial introduction scenarios as unsampled population scenarios, with no major loss in classification accuracy.

We found that posterior probability distributions were often flat when errors of classification were observed, resulting in large $95 \% \mathrm{CI}$ and low levels of confidence in the results obtained. Such flat posterior probabilities should, therefore, be interpreted with caution, as they may indicate errors in parameter priors and/or model specification. The comparison between observed summary statistics and simulated statistic distributions may also be used to detect such errors (Pascual et al., 2007). In practice, one could advise ABC users to (i) choose broad support of priors (that is region of the prior with positive probabilities) to ensure that it includes the 'true values' of parameters, (ii) be sceptical about prior distributions and/or model specifications when obtaining low maximal probabilities with large $95 \%$ CI and (iii) compare the results obtained with and without the unsampled and the inverse-serial scenarios to be able to detect error in scenario specifications.

The evaluation of the ABC method presented here was subject to several limitations. This study deals with a simple biological invasion situation, in which only two invading populations and a single source are considered. Although this setting can be used as the basis for retracing more complex multipopulational introduction histories (for example Miller et al., 2005), the ABC method implemented in DIYABC can handle complex scenarios of introduction routes with a large number of populations directly (see Cornuet et al., 2008, for methodological details and illustrations). For instance, admixture can be modeled in DIYABC and the assumption of a single source can easily be relaxed by considering two populations that diverged some generations ago and are the sources of the introduced populations. We also assumed that there was no migration between invading populations and no recurrent introductions into each invading population. Although considering migration is crucial in some cases, the absence of migration may be a reasonable approximation in many circumstances, particularly when the invading populations are geographically distant from the original source population and from each other (as for the Italian and Central European outbreaks of WCR or the populations of Drosophila subobscura introduced into North and South America, Pascual et al., 2007). Recurrent introductions from the same source population into an invading population, although not probable in the case of recent introductions, would probably be equivalent to increasing propagule size and would, thus, not be genetically distinguishable from a single introduction with a larger propagule size (although this remains to be carefully tested). Finally, it would be worth evaluating the ABC method for estimating probabilities of introduction routes for datasets including other genetic markers such as DNA sequences, SNPs or AFLP markers.

\section{Acknowledgements}

We thank Christian Robert and Jean-Michel Marin, who helped us to compare posterior probability estimators, Filipe Santos and Sylvain Piry for assistance with computer cluster installation and Aurélie Blin and Pascal Chavigny for technical assistance. This work was funded by the French National Research Agency (ANR) through project grants ANR-06-BDIV-008-01 to TG and AE and ANR-BLAN-0196-01 to J-MC and AE.

\section{References}

Beaumont M (2008). Joint determination of topology, divergence time, and immigration in population trees. In: Matsumura S, Forster P, Renfrew C (eds). Simulations, Genetics and Human Prehistory. McDonald Institute for Archaeological Research: Cambridge. pp 135-154.

Beaumont MA, Zhang WY, Balding DJ (2002). Approximate Bayesian computation in population genetics. Genetics 162: 2025-2035.

Ciosi M, Miller NJ, Kim KS, Giordano R, Estoup A, Guillemaud $\mathrm{T}$ (2008). Invasion of Europe by the western corn rootworm, Diabrotica virgifera virgifera: multiple transatlantic introductions and various patterns of reduced genetic diversity. Mol Ecol 17: 3614-3627.

Cornuet JM, Santos F, Beaumont MA, Robert CP, Marin J-M, Balding DJ et al. (2008). Inferring population history with DIYABC: a user-friendly approach to approximate Bayesian computation. Bioinformatics 24: 2713-2719.

Dlugosch KM, Parker IM (2008). Founding events in species invasions: genetic variation, adaptive evolution, and the role of multiple introductions. Mol Ecol 17: 431-449.

Estoup A, Beaumont M, Sennedot F, Moritz C, Cornuet JM (2004). Genetic analysis of complex demographic scenarios: spatially expanding populations of the cane toad, Bufo marinus. Evolution 58: 2021-2036.

Estoup A, Jarne P, Cornuet JM (2002). Homoplasy and mutation model at microsatellite loci and their consequences for population genetics analysis. Mol Ecol 11: 1591-1604.

Fagundes NJR, Ray N, Beaumont MA, Neuenschwander S, Salzano F, Bonatto SL et al. (2007). Statistical evaluation of alternative models of human evolution. Proc Natl Acad Sci USA 104: 17614-17619.

Fawcett T (2006). An introduction to ROC analysis. Pattern Recognit Lett 27: 861-874.

Gaggiotti OE, Brooks SP, Amos W, Harwood J (2004). Combining demographic, environmental and genetic data to test hypotheses about colonization events in metapopulations. Mol Ecol 13: 811-825.

Garza JC, Williamson EG (2001). Detection of reduction in population size using data from microsatellite loci. Mol Ecol 10: $305-318$.

Goldstein DB, Roemer GW, Smith DA, Reich DE, Bergman A, Wayne RK (1999). The use of microsatellite variation to infer population structure and demographic history in a natural model system. Genetics 151: 797-801.

Hudson RR (1990). Gene genealogies under the coalescent process. In: Futuyma DJ, Antonovics J (eds). Oxford Survey in Evolutionary Biology. Vol. 7, Oxford University Press: Oxford pp 1-44.

Kim KS, Sappington TW (2005). Genetic structuring of western corn rootworm (Coleoptera : Chrysomelidae) populations in the United States based on microsatellite loci analysis. Environ Entomol 34: 494-503.

Kingman JFC (1982). The coalescent. Stochastic Process Appl 13: 235-248. 
Kolbe JJ, Glor RE, Schettino LRG, Lara AC, Larson A, Losos JB (2004). Genetic variation increases during biological invasion by a Cuban lizard. Nature 431: 177-181.

Miller N, Estoup A, Toepfer S, Bourguet D, Lapchin L, Derridj S et al. (2005). Multiple transatlantic introductions of the western corn rootworm. Science 310: 992.

Nei M (1987). Molecular Evolutionary Genetics. Columbia University Press: New York.

Nordborg M (2001). Coalescent theory. In: Balding DJ, Bishop M, Cannings C (eds). Handbook of Statitical Genetics. John Wiley \& Sons, Ltd.: Chichester, England. pp 179-212.

Pascual M, Chapuis MP, Mestres F, Balanya J, Huey RB, Gilchrist GW et al. (2007). Introduction history of Drosophila subobscura in the New World: a microsatellite-based survey using ABC methods. Mol Ecol 16: 3069-3083.

Rannala B, Mountain JL (1997). Detecting immigration by using multilocus genotypes. Proc Natl Acad Sci USA 94: 9197-9201.

Roman J (2006). Diluting the founder effect: cryptic invasions expand a marine invader's range. Proc $R$ Soc B-Biol Sci 273: 2453-2459.
Saltonstall K (2002). Cryptic invasion by a non-native genotype of the common reed, Phragmites australis, into North America. Proc Natl Acad Sci USA 99: 2445-2449.

Suarez AV, Holway DA, Case TJ (2001). Patterns of spread in biological invasions dominated by long-distance jump dispersal: insights from Argentine ants. Proc Natl Acad Sci USA 98: 1095-1100.

Terrel GR, Scott DW (1992). Variable kernel density estimation. Ann Stat 20: 1236-1265.

Toepfer S, Kuhlmann U (2005). Natural mortality factors acting on western corn rootworm populations: a comparison between the United States and Central Europe. In: Vidal S, Kuhlmann U, Edwards CR (eds). Western Corn Rootworm: Ecology and Management. CABI Publishing: Wallingford, UK. pp 95-119.

Voisin M, Engel CR, Viard F (2005). Differential shuffling of native genetic diversity across introduced regions in a brown alga: aquaculture vs. maritime traffic effects. Proc Natl Acad Sci USA 102: 5432-5437.

Weir BS, Cockerham C (1984). Estimating F-statistics for the analysis of population structure. Evolution 38: 1358-1370.

Supplementary Information accompanies the paper on Heredity website (http://www.nature.com/hdy) 